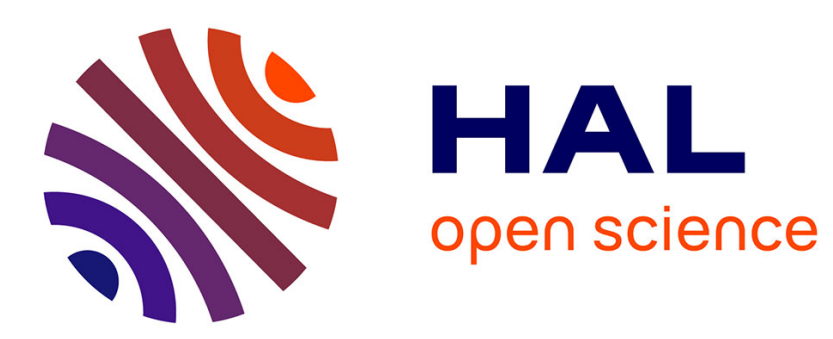

\title{
Goal-oriented error estimation based on equilibrated-flux reconstruction for finite element approximations of elliptic problems
}

Igor Mozolevski, Serge Prudhomme

\section{To cite this version:}

Igor Mozolevski, Serge Prudhomme. Goal-oriented error estimation based on equilibrated-flux reconstruction for finite element approximations of elliptic problems. 2014. hal-00985971

\section{HAL Id: hal-00985971 \\ https://hal.science/hal-00985971}

Preprint submitted on 5 May 2014

HAL is a multi-disciplinary open access archive for the deposit and dissemination of scientific research documents, whether they are published or not. The documents may come from teaching and research institutions in France or abroad, or from public or private research centers.
L'archive ouverte pluridisciplinaire HAL, est destinée au dépôt et à la diffusion de documents scientifiques de niveau recherche, publiés ou non, émanant des établissements d'enseignement et de recherche français ou étrangers, des laboratoires publics ou privés. 


\title{
Goal-oriented error estimation based on equilibrated-flux reconstruction for finite element approximations of elliptic problems
}

\author{
Igor Mozolevskia ${ }^{\mathrm{a}, *}$, Serge Prudhomme ${ }^{\mathrm{b}}$ \\ ${ }^{a}$ Federal University of Santa Catarina (UFSC) \\ Campus Universitário, Trindade, 88040-900 Florianópolis, SC, Brazil \\ ${ }^{b}$ Department of Mathematics and Industrial Engineering \\ École Polytechnique de Montréal \\ C.P. 6079, Succ. Centre-ville, Montréal, Québec H3C 3A7, Canada
}

\begin{abstract}
We propose an approach for goal-oriented error estimation in finite element approximations of second-order elliptic problems that combines the dualweighted residual method and equilibrated-flux reconstruction methods for the primal and dual problems. The objective is to be able to consider discretization schemes for the dual solution that may be different from those used for the primal solution. It is only assumed here that the discretization methods come with a priori error estimates and an equilibrated-flux reconstruction algorithm. A high-order discontinuous Galerkin (dG) method is actually the preferred choice for the approximation of the dual solution thanks to its flexibility and straightforward construction of equilibrated fluxes. One contribution of the paper is to show how the order of the $\mathrm{dG}$ method for asymptotic exactness of the proposed estimator can be chosen in the cases where a conforming finite element method, a dG method, or a mixed RaviartThomas method are used for the solution of the primal problem. Numerical experiments are also presented to illustrate the performance and convergence of the error estimates in quantities of interest with respect to the mesh size.
\end{abstract}

Keywords: Finite element method, goal-oriented estimates, quantity of interest, dual problem, asymptotically-exact error estimates

\footnotetext{
${ }^{*}$ Corresponding author. Tel/Fax: +55 (48) 3721-9232

Email addresses: igor.mozolevski@ufsc.br (Igor Mozolevski), serge.prudhomme@polymtl.ca (Serge Prudhomme)
} 


\section{Introduction}

A variety of finite element discretization schemes, such as the mixed finite element methods, the non-conforming finite element methods, or discontinuous Galerkin ( $\mathrm{d} G$ ) finite element methods, have been developed during the past decades in order to provide better approximation properties than those offered by the classical finite element method depending on the type of partial differential equations at hand. These methods have become increasingly popular and are now widely used for solving problems of various interest in engineering and sciences. At the same time, the necessity to obtain accurate finite element approximations to given boundaryvalue problems has stimulated the development of a posteriori error estimators that provide fully computable, reliable, and efficient error bounds in terms of the problem data and the finite element approximation. In the case of standard conforming finite element schemes, a posteriori error estimates with respect to global energy norms are indeed well-established, see e.g. $[52,4,7,8,43,31,50,53]$. A posteriori error estimation for nonconforming finite element methods with application to second-order elliptic problems has recently seen significant progress and is still the subject of sustained research efforts, see e.g. [9, 34, 51, 55, 33, 1, 18, 42, 41, 27, 24] for dG methods and $[16,35,2,17,37]$ for mixed finite element methods. We also refer the reader to $[36,18,3,28,22]$ and references therein for the presentation of unifying frameworks on the topic.

In practical applications, end users are however interested in error estimates in some specific features of the true solution. These so-called quantities of interest, either local or global, are represented as functionals defined on the vector space of trial solutions of the boundary-value problem. Error estimation with respect to such functionals is usually referred to as goal-oriented error estimation. The key ingredient for goal-oriented error estimation is the formulation of an auxiliary problem, the dual problem to the primal problem, whose solution provides necessary information for reliable estimates of the error in the goal functional. Several strategies for goal-oriented error estimation have been proposed in the case of elliptic problems: goal-oriented error estimates based on energy norm of the errors in the primal and dual solutions were introduced in $[48,45,46,49]$ and further developed by various authors, see for example $[4,5]$, and references therein, error estimates using 
the dual-weighted residual method were proposed in $[25,10,8]$; functional a posteriori error estimates were developed in [43, 50]; estimates based on the gradient-recovery method were considered in [39, 38, 47, 44, 40]; finally, goal-oriented estimates for discontinuous Galerkin methods in the case of second-order elliptic problems were derived in [32].

The general approach to obtain goal-oriented error estimates consists, on one hand, in deriving an error representation involving the residual functional and the exact dual (adjoint) solution, and, on the other hand, in constructing a sufficiently accurate approximation of the adjoint solution in order to obtain a fully computable and reliable error estimate along with local refinement indicators. In the case of the classical conforming finite element method, such approximation to the adjoint solution is usually calculated on a refinement of the mesh used for the primal solution, with the same polynomial degree, or, preferably, on the same mesh, but with a higher polynomial degree.

In this paper, we propose an alternative approach to goal-oriented estimation by considering an error representation that does not uses the orthogonality property and is amenable to different types of discretization of the primal and dual problems. We only suppose here that the method used to discretize the primal problem produces piecewise polynomial solutions from which equilibrated fluxes can be reconstructed (this is not a restrictive property) and satisfies standard a priori error estimates in the $L^{2}$ and energy norms. Using an error representation similar to the one used in the dual-weighted residual method, we use the flux-equilibration technique to decompose the error into a computable error estimator and a higher-order remainder. It is well known, see e.g. [5], that in order to obtain an efficient error estimator (effectivity indices remain close to unity), the dual problem must be approximated using a higher-order approximation than the one used in the finite element approximation of the primary problem. We propose in this paper, in order to approximate the dual solution, that a high-order discontinuous Galerkin method be used and applied on the same mesh as that used for the discretization of the primal problem. The choice of the $\mathrm{dG}$ method seems natural owing to its flexibility in using non-uniform high-order polynomials. Furthermore, the $\mathrm{dG}$ method benefits from the fact that it is locally conservative; it implies that the construction of equilibrated fluxes, needed for the evaluation of the proposed error estimator, is rather straightforward, see e.g. [36, 26, 19, 12]. Finally, we show that, depending on the approximation properties of the primal method and problem data, the order of the $\mathrm{dG}$ method, used to solve the dual problem, can be chosen in such a 
way that the respective error estimator is asymptotically exact.

The paper is organized as follows. Section 2 introduces the model (primal) problem, the corresponding dual problem, and preliminary notation for goal-oriented error estimation. Section 3 presents a suitable error representation for the goal functional based on the use of equilibrated fluxes in terms of the finite element solutions to the primal and dual problems. We also show how the error representation can be decomposed into a fully computable error estimator and a higher-order term, which can be evaluated using 1) a priori estimates with respect to the primal and dual discretization schemes and 2) methods for reconstruction of equilibrated fluxes. We briefly introduce in Section 4 the symmetric version of the dG method. We then show how the order of the $\mathrm{dG}$ method, used for the discretization of the dual problem, can be chosen to guarantee asymptotical exactness of the error estimator when discretizing the primal problem by high-order $\mathrm{dG}$ methods, conforming finite element methods, and mixed finite element methods, as described in Sections 5, 6, and 7, respectively. Finally, we present some numerical examples to demonstrate the performance of the estimators in Section 8 before concluding in Section 9.

\section{Model problem}

In this work, we are primarily interested in general linear boundary-value problems defined in terms of elliptic second-order partial differential equations, but the results could be extended to more general situations. For the sake of clarity in the presentation, we shall restrict ourselves to a simple model problem. Let $\Omega \in \mathbb{R}^{2}$ be a polygonal domain with boundary $\partial \Omega$. We consider the homogeneous Dirichlet boundary-value problem whose solution $u$ satisfies

$$
\begin{aligned}
-\nabla \cdot(D \nabla u)=f & \text { in } \Omega, \\
u=0 & \text { on } \partial \Omega .
\end{aligned}
$$

where coefficient $D=D(\mathbf{x}), \mathbf{x} \in \Omega$, is a piecewise constant strictly positive scalar function and $f \in L^{2}(\Omega)$. The weak formulation of the problem reads:

$$
\text { Find } u \in H_{0}^{1}(\Omega) \text { such that } B(u, v)=F(v), \forall v \in H_{0}^{1}(\Omega)
$$


where $B$ and $F$ are the bilinear and linear forms on $H_{0}^{1}(\Omega)$, respectively, defined as:

$$
\begin{aligned}
& B(u, v)=\int_{\Omega} D \nabla u \cdot \nabla v, \\
& F(v)=\int_{\Omega} f v .
\end{aligned}
$$

We shall suppose here that the elliptic regularity property holds true for the model problem; i.e. there exist a unique weak solution $u \in H^{2}(\Omega)$ to $(2)$ and a constant $C>0$ that only depends on $\Omega$, such that

$$
\|u\|_{H^{2}(\Omega)} \leq C\|f\|_{L^{2}(\Omega)} .
$$

Note that the conditions for elliptic regularity are ensured if the polygonal domain $\Omega$ is assumed convex; in more general cases, the regularity of the solution usually depends on the internal angles between the boundary edges of $\Omega$, see [30]. In the remainder of the paper, we will denote by $C$ a generic constant that may depend on the problem data and other model parameters. This dependence will be made explicit in the text when necessary.

We assume that we shall be interested in the linear goal functional $Q$ defined on $L^{2}(\Omega)$ such that:

$$
Q(v)=\int_{\Omega} q v
$$

where $q \in L^{2}(\Omega)$ denotes the Riesz representer of $Q$. We introduce the corresponding dual problem, in strong form as:

$$
\begin{aligned}
-\nabla \cdot(D \nabla p)=q & \text { in } \Omega, \\
p=0 & \text { on } \partial \Omega,
\end{aligned}
$$

and in weak form as:

$$
\text { Find } p \in H_{0}^{1}(\Omega) \text { such that } B(v, p)=Q(v), \forall v \in H_{0}^{1}(\Omega) \text {. }
$$

We again emphasize that, owing to the elliptic regularity and the fact that $q \in L^{2}(\Omega)$, we have that $p \in H^{2}(\Omega)$. It is also straightforward to show from (2) and (8) that

$$
F(p)=B(u, p)=Q(u)
$$


Let $\mathcal{T}_{h}, h>0$, be a family of shape-regular triangular meshes on $\Omega$, see e.g. [14], where $h=\max _{T \in \mathcal{T}_{h}} h(T)$ denotes the mesh size and $h(T)$ is the diameter of mesh element $T$. We assume here that the edges of two neighboring elements will perfectly match with each other, in the sense that the meshes will be free of so-called hanging nodes. For a triangular mesh $\mathcal{T}_{h}$, we denote by $\mathcal{E}_{h}$ the set of all edges in the mesh; $\mathcal{E}_{h}$ can be further decomposed into the set of interior edges $\mathcal{E}_{h}^{\mathrm{i}}$ and the set of boundary edges $\mathcal{E}_{h}^{\partial}$, that is $\mathcal{E}_{h}=\mathcal{E}_{h}^{\mathrm{i}} \cup \mathcal{E}_{h}^{\partial}$. By definition, an edge $E \in \mathcal{E}_{h}$ is said to be an interior edge if there exist two triangles $T^{-}, T^{+} \in \mathcal{T}_{h}$ such that $\bar{E}=\bar{T}^{-} \cap \bar{T}^{+}$and is said to be a boundary edge if there exists one and only one triangle $T \in \mathcal{T}_{h}$ such that $\bar{E}=\bar{T} \cap \partial \Omega$. For a polygonal domain $\Omega$, we have $\partial \Omega=\cup_{E \in \mathcal{E}_{h}^{\partial}} \bar{E}$. For any function $v \in L^{2}(\Omega)$, assumed sufficiently smooth to admit a trace on all $E \in \mathcal{E}_{h}$ (possibly different on either side of an edge $E \in \mathcal{E}_{h}^{\text {i }}$ ), let us denote the jump of $v$ along $E$ by

$$
\begin{array}{ll}
{[[v]]:=\left.v_{T^{-}}\right|_{E}-\left.v_{T^{+}}\right|_{E},} & E \in \mathcal{E}_{h}^{\mathrm{i}}, \\
{[[v]]:=\left.v\right|_{E},} & E \in \mathcal{E}_{h}^{\partial},
\end{array}
$$

where $\left.v^{ \pm}\right|_{E}$ denotes the trace on the respective side of $E$. For $E \in \mathcal{E}_{h}^{\mathrm{i}}$, we denote by $\mathbf{n}_{E}$ the unit normal vector to $E$ pointing from $T^{-}$toward $T^{+}$, whereas for $E \in \mathcal{E}_{h}^{\partial}$, we set $\mathbf{n}_{E}$ equal to the unit external normal $\mathbf{n}$ to $\partial \Omega$. The orientation of $\mathbf{n}_{E}$ for interior faces is chosen in accordance with the definition of the jump; in such a case, the arbitrariness in the choice of $T^{-}$ and $T^{+}$in the definition of the interior edge is irrelevant. We also introduce the standard arithmetic average of $v$ along $E$ as

$$
\begin{array}{ll}
\{\{v\}\}=\frac{1}{2}\left(\left.v_{T^{-}}\right|_{E}+\left.v_{T^{+}}\right|_{E}\right), & E \in \mathcal{E}_{h}^{\mathrm{i}}, \\
\{\{v\}\}=[[v]]=\left.v\right|_{E}, & E \in \mathcal{E}_{h}^{\partial} .
\end{array}
$$

Finally, let $\mathbb{P}_{k}(T)$ denote the vector space of polynomials on $T$ of degree $k$ or less. We then introduce the finite element space $\mathcal{V}_{h}^{k}$ as:

$$
\mathcal{V}_{h}^{k}:=\left\{v_{h} \in L^{2}(\Omega):\left.v_{h}\right|_{T} \in \mathbb{P}_{k}(T), \forall T \in \mathcal{T}_{h}\right\} .
$$

For a given mesh $\mathcal{T}_{h}$, let us denote by $u_{h} \in \mathcal{V}_{h}^{k}$ an arbitrary approximation of the exact solution $u$ to the primal problem (2). The objective in this paper is to study the approximation error in the goal functional

$$
\mathcal{E}=Q(u)-Q\left(u_{h}\right)
$$


At this stage, it is not necessary to specify the numerical scheme with which the approximate solution is obtained. Since the goal functional considered here is assumed linear and continuous on $L^{2}(\Omega)$, it is sufficient to suppose that $u_{h}$ converges to the exact solution $u$ in $L^{2}(\Omega)$ in order for $Q\left(u_{h}\right)$ to be a reasonable approximation of $Q(u)$.

We will make use of the following definition in the remainder of the paper:

Definition 1. The error $\mathcal{E}=Q(u)-Q\left(u_{h}\right)$ in the goal functional is said to be properly of order $l>0$ with respect to the discrete solution $u_{h}$ if there exist constants $\underline{C}>0$ and $\bar{C}>0$, that depend on $u$ and $Q$ only, such that

$$
\underline{C} h^{l} \leq\left|Q(u)-Q\left(u_{h}\right)\right| \leq \bar{C} h^{l}
$$

for sufficiently small $h>0$.

We propose below an asymptotically exact estimator of the error in the goal functional (13) based on the condition that the error be properly of order $l>0$ with respect to a discrete solution family $\left\{u_{h}\right\}_{h>0}$.

\section{Goal-oriented error representation}

In this section, we briefly review the notion of equilibrated fluxes and present a goal-oriented error representation in terms of reconstructed equilibration fluxes. We first recall the space $\mathbf{H}(\operatorname{div}, \Omega)$ of vector-valued functions

$$
\mathbf{H}(\operatorname{div}, \Omega)=\left\{\mathbf{t} \in\left[L^{2}(\Omega)\right]^{2}: \nabla \cdot \mathbf{t} \in L^{2}(\Omega)\right\},
$$

and the Raviart-Thomas finite element space of order $m \in \mathbb{N}_{0}$ :

$$
\mathbf{R T}_{m}\left(\mathcal{T}_{h}\right)=\left\{\mathbf{t}_{h} \in \mathbf{H}(\operatorname{div}, \Omega):\left.\mathbf{t}_{h}\right|_{T} \in\left[\mathbb{P}_{m}(T)\right]^{2}+\mathbf{x} \mathbb{P}_{m}(T), \forall T \in \mathcal{T}_{h}\right\}
$$

Definition 2. Let $u_{h} \in \mathcal{V}_{h}^{k}, k \in \mathbb{N}_{0}$, and let $l=\max \{0, k-1\}$. Let $\pi_{h}^{l}$ : $L^{2}(\Omega) \rightarrow \mathcal{V}_{h}^{l}$ denote the $L^{2}$-orthogonal projection operator. A vector $\mathbf{t}_{h}\left(u_{h}\right) \in$ $\mathbf{R T}_{l}\left(\mathcal{T}_{h}\right)$, reconstructed from the approximation $u_{h}$, is said to be an equilibrated flux with respect to Problem (1) if

$$
\nabla \cdot \mathbf{t}_{h}\left(u_{h}\right)=\pi_{h}^{l}(f) .
$$

Note that we will use the notation $\mathbf{t}_{h}^{l}\left(u_{h}\right)=\mathbf{t}_{h}\left(u_{h}\right)$ when the order of the Raviart-Thomas space in which the flux is reconstructed needs to be mentioned explicitly. 
The concept of equilibrated fluxes has been widely used to construct accurate a posteriori error estimates for conforming finite element approximations since such estimates are exempt of unknown constants, see for example $[4,11]$. In fact, various flux reconstruction techniques have been developed over the years in the case of elliptic problems and some of the techniques have been extended to non-conforming conservative methods [36, 26, 12], to first-order conforming finite element method [13], or to the finite volume and finite difference methods [54].

We propose here to investigate how reconstructed equilibrated fluxes could be used in goal-oriented error estimation. We start by deriving the following error representation with respect to the goal functional, using (9), the dual solution $p$ to problem (7), and integration by parts,

$$
\begin{aligned}
\mathcal{E} & =Q(u)-Q\left(u_{h}\right)=F(p)-Q\left(u_{h}\right) \\
& =\int_{\Omega} f p+\int_{\Omega}(\nabla \cdot D \nabla p) u_{h} \\
& =\sum_{T \in \mathcal{T}_{h}}\left[\int_{T}\left(f p-D \nabla u_{h} \cdot \nabla p\right)+\int_{\partial T}(\mathbf{n} \cdot D \nabla p) u_{h}\right] .
\end{aligned}
$$

Introducing the jump (10) in $u_{h}$ across edge $E$, the sum of the edge integrals can be recast as:

$$
\mathcal{E}=\sum_{T \in \mathcal{T}_{h}} \int_{T}\left(f p-D \nabla u_{h} \cdot \nabla p\right)+\sum_{E \in \mathcal{E}_{h}} \int_{E}\left(\mathbf{n}_{E} \cdot D \nabla p\right)\left[\left[u_{h}\right]\right] .
$$

Note that for an arbitrary $\mathbf{t}_{h} \in \mathbf{R} \mathbf{T}_{l}\left(\mathcal{T}_{h}\right)$, above relation can be rewritten, by simple addition and subtraction of the term $\mathbf{t}_{h} \cdot \nabla p$, as:

$$
\begin{aligned}
\mathcal{E}=\sum_{T \in \mathcal{T}_{h}} \int_{T}\left(f p+\mathbf{t}_{h} \cdot \nabla p\right)+\sum_{T \in \mathcal{T}_{h}} \int_{T}\left(-\mathbf{t}_{h}-D \nabla u_{h}\right) \cdot \nabla p \\
+\sum_{E \in \mathcal{E}_{h}} \int_{E}\left(\mathbf{n}_{E} \cdot D \nabla p\right)\left[\left[u_{h}\right]\right] \\
=\sum_{T \in \mathcal{T}_{h}}\left[\int_{T}\left(f-\nabla \cdot \mathbf{t}_{h}\right) p+\sum_{E \in \partial T} \int_{E}\left(\mathbf{t}_{h} \cdot \mathbf{n}\right) p\right] \\
+\sum_{T \in \mathcal{T}_{h}} \int_{T}\left(-\mathbf{t}_{h}-D \nabla u_{h}\right) \cdot \nabla p+\sum_{E \in \mathcal{E}_{h}} \int_{E}\left(\mathbf{n}_{E} \cdot D \nabla p\right)\left[\left[u_{h}\right]\right] .
\end{aligned}
$$


Since, owing to the homogeneous Dirichlet boundary condition on the dual solution and the continuity of the normal component of $\mathbf{t}_{h}$,

$$
\sum_{T \in \mathcal{T}_{h}}\left[\sum_{E \in \partial T} \int_{E}\left(\mathbf{t}_{h} \cdot \mathbf{n}\right) p\right]=\sum_{E \in \mathcal{E}_{h}}\left[\left[\mathbf{t}_{h} \cdot \mathbf{n}\right]\right] p=0
$$

we then obtain:

$$
\begin{aligned}
\mathcal{E}=\sum_{T \in \mathcal{T}_{h}} & \int_{T}\left(f-\nabla \cdot \mathbf{t}_{h}\right) p-\sum_{T \in \mathcal{T}_{h}} \int_{T}\left(\mathbf{t}_{h}+D \nabla u_{h}\right) \cdot \nabla p \\
& +\sum_{E \in \mathcal{E}_{h}} \int_{E}\left(\mathbf{n}_{E} \cdot D \nabla p\right)\left[\left[u_{h}\right]\right] .
\end{aligned}
$$

We are now ready to establish the following error representation in terms of the goal functional.

Theorem 1. Let $u_{h} \in \mathcal{V}_{h}^{k}, k \in \mathbb{N}_{0}$, be an approximation of the solution $u \in H_{0}^{1}(\Omega)$ to the primal problem (1) and let $p_{h} \in \mathcal{V}_{h}^{m}, m>k$, be an approximation of the solution $p \in H_{0}^{1}(\Omega) \cap H^{2}(\Omega)$ to the dual problem (7). Suppose that $u_{h}$ and $p_{h}$ admit reconstructed equilibrated fluxes $\mathbf{t}_{h}\left(u_{h}\right) \in \mathbf{R T}_{l}\left(\mathcal{T}_{h}\right)$, $l=\max \{0, k-1\}$ and $\mathbf{t}_{h}\left(p_{h}\right) \in \mathbf{R} \mathbf{T}_{m-1}$, respectively. Then the error in the goal functional $Q$ can be represented as

$$
\mathcal{E}=Q(u)-Q\left(u_{h}\right)=\eta\left(u_{h}, p_{h} ; \mathbf{t}_{h}\left(u_{h}\right), \mathbf{t}_{h}\left(p_{h}\right)\right)+\mathcal{R}\left(u, p ; u_{h}, p_{h}\right),
$$

where the error estimator $\eta$ is defined as:

$$
\begin{aligned}
\eta & =\eta\left(u_{h}, p_{h} ; \mathbf{t}_{h}\left(u_{h}\right), \mathbf{t}_{h}\left(p_{h}\right)\right) \\
& =\sum_{T \in \mathcal{T}_{h}} \int_{T}\left(f-\pi_{h}^{l}(f)\right) p_{h}+\sum_{T \in \mathcal{T}_{h}} \int_{T}\left(\mathbf{t}_{h}\left(u_{h}\right)+D \nabla u_{h}\right) \cdot D^{-1} \mathbf{t}_{h}\left(p_{h}\right) \\
& -\sum_{E \in \mathcal{E}_{h}} \int_{E}\left(\mathbf{n}_{E} \cdot \mathbf{t}_{h}\left(p_{h}\right)\right)\left[\left[u_{h}\right]\right],
\end{aligned}
$$

and the remainder term is:

$$
\begin{aligned}
\mathcal{R}\left(u, p, f ; u_{h}, p_{h}\right) & =\sum_{T \in \mathcal{T}_{h}} \int_{T}\left(f-\pi_{h}^{l}(f)\right)\left(p-p_{h}\right) \\
& -\sum_{T \in \mathcal{T}_{h}} \int_{T}\left(\mathbf{t}_{h}\left(u_{h}\right)+D \nabla u_{h}\right) \cdot D^{-1}\left(D \nabla p+\mathbf{t}_{h}\left(p_{h}\right)\right) \\
& +\sum_{E \in \mathcal{E}_{h}} \int_{E}\left(\mathbf{n}_{E} \cdot\left(D \nabla p+\mathbf{t}_{h}\left(p_{h}\right)\right)\right)\left[\left[u_{h}\right]\right] .
\end{aligned}
$$


Remark 1. The error estimator $\eta$ can be easily decomposed into a sum of local element error contributions. Indeed, let us note that

$$
\sum_{E \in \mathcal{E}_{h}} \int_{E} \mathbf{t}_{h}\left(p_{h}\right) \cdot \mathbf{n}\left[\left[u_{h}\right]\right]=\sum_{T \in \mathcal{T}_{h}} \sum_{E \in \partial T} \int_{E} \chi_{\mathcal{E}_{h}}(E)\left(\mathbf{n} \cdot \mathbf{t}_{h}\left(p_{h}\right)\right)\left[\left[u_{h}\right]\right],
$$

where $\chi_{\mathcal{E}_{h}}$ denotes the edge indicator function on $\mathcal{E}_{h}$, that is $\chi_{\mathcal{E}_{h}}(E)=1 / 2$, if $E \in \mathcal{E}_{h}^{\mathrm{i}}$, and $\chi_{\mathcal{E}_{h}}=1$, if $E \in \mathcal{E}_{h}^{\partial}$. Therefore, in that case, the decomposition would take the form:

$$
\eta=\sum_{T \in \mathcal{T}_{h}} \eta_{T}(T)=\sum_{T \in \mathcal{T}_{h}} \eta_{\mathcal{O}}(T)+\eta_{\nabla}(T)+\eta_{\mathcal{H}}(T)
$$

with

$$
\begin{aligned}
& \eta_{\mathcal{O}}(T)=\int_{T}\left(f-\pi_{h}^{l}(f)\right) p_{h} \\
& \eta_{\nabla}(T)=\int_{T}\left(\mathbf{t}_{h}\left(u_{h}\right)+D \nabla u_{h}\right) \cdot D^{-1} \mathbf{t}_{h}\left(p_{h}\right) \\
& \eta_{\mathcal{H}}(T)=\sum_{E \in \partial T} \int_{E} \chi_{\mathcal{E}_{h}}(E)\left[\left[u_{h}\right]\right]\left(\mathbf{n}_{E} \cdot \mathbf{t}_{h}\left(p_{h}\right)\right)
\end{aligned}
$$

Here $\eta_{\mathcal{O}}(T)$ represents the data oscillation in element $T$ with respect to the primal problem, weighted by the dual approximate solution; the flux estimator $\eta_{\nabla}(T)$ measures the deviation of the discrete gradient $-D \nabla u_{h}$ from the reconstructed flux $\mathbf{t}_{h}\left(u_{h}\right) \in \mathbf{H}\left(\right.$ div, $\left.\mathcal{T}_{h}\right)$; and $\eta_{\mathcal{H}}(T)$ measures the deviation of $u_{h}$ from $H_{0}^{1}(\Omega)$, both weighted by the reconstructed equilibrated flux of the dual approximate solution. Note that the error estimator $\eta$ is fully computable once the flux reconstructions for $\mathbf{t}_{h}\left(u_{h}\right)$ and $\mathbf{t}_{h}\left(p_{h}\right)$ are established.

Remark 2. Depending on the properties of the data in the primal problem, the error estimator (21) may take a simpler form; e.g. the oscillation term disappears for $f \in \mathcal{V}_{h}^{l}$.

Remark 3. Depending on the discretization method used, the finite element approximation $u_{h}$ may weakly (or strongly) satisfy additional continuity properties along the interior edges and specific boundary conditions on the boundary edges of the mesh. Values of the different components of the error estimator will thus strongly depend on the choice of the numerical method used in the approximation of the primal problem. For example, if conforming finite element methods are used, $\eta_{\mathcal{H}}=0$. 
In the remainder of the paper, we show how the error representation (21) can provide an asymptotically exact goal-oriented estimator of the error in approximations of the primal problem. One crucial ingredient of the estimator is the choice of the finite element method for the approximation of the dual solution. If one prefers to keep the same mesh to solve the dual problem as that used for the primal finite element solution, while retaining an efficient error estimator, higher-order methods should be employed, see e.g. [5]. Furthermore, it is desirable that the discretization method for the dual problem allows for computationally inexpensive equilibrated-flux reconstruction. Based on these facts, we propose here to use a high-order discontinuous Galerkin discretization method to approximate the dual solution. Advantages of the $\mathrm{dG}$ method are that high-order elements in any space dimension can be easily implemented and that it is locally conservative, which yields in a straightforward manner equilibrated fluxes. In fact, it has been shown to provide cheap and local reconstruction algorithms [35, 26, 19, 12].

\section{The dG method}

We briefly introduce in this section the symmetric interior penalty $\mathrm{dG}$ method and present some basic results on the stability and approximation

properties of the method. We first introduce the bilinear form $B_{h}: \mathcal{V}_{h}^{k} \times \mathcal{V}_{h}^{k} \rightarrow$ $\mathbb{R}$ as

$$
\begin{aligned}
B_{h}\left(u_{h}, v_{h}\right) & =\sum_{T \in \mathcal{T}_{h}} \int_{T} D \nabla u_{h} \cdot \nabla v_{h}-\sum_{E \in \mathcal{E}_{h}} \int_{E}\left\{\left\{\mathbf{n} \cdot D \nabla u_{h}\right\}\right\}\left[\left[v_{h}\right]\right] \\
& +\sum_{E \in \mathcal{E}_{h}} \int_{E}\left(-\left\{\left\{\mathbf{n} \cdot D \nabla v_{h}\right\}\right\}+\gamma_{E}\left[\left[v_{h}\right]\right]\right)\left[\left[u_{h}\right]\right],
\end{aligned}
$$

where $\gamma_{E}>0$ denotes the interior penalty parameter to be specified later. For the primal boundary value problem (1), the symmetric interior penalty $\mathrm{dG}$ method is defined as follows (see e.g. [20]):

$$
\text { Find } u_{h} \in \mathcal{V}_{h}^{k} \text { such that } B_{h}\left(u_{h}, v_{h}\right)=F\left(v_{h}\right), \forall v_{h} \in \mathcal{V}_{h}^{k} \text {. }
$$

Since symmetric dG method is adjoint consistent, the corresponding dual problem reads:

$$
\text { Find } p_{h} \in \mathcal{V}_{h}^{m} \text { such that } B_{h}\left(v_{h}, p_{h}\right)=Q\left(v_{h}\right), \forall v_{h} \in \mathcal{V}_{h}^{m} \text {. }
$$


For completeness, we present below some basic analytical results referring to the symmetric interior penalty $\mathrm{d} G$ method; owing to the symmetry of the method and of the primal and dual problems, we can limit the analysis to the primal problem only. Firstly, let us note that, for sufficiently large penalty parameters, problem (26) has a unique solution, see e.g. [20]. We introduce here a computable lower bound $\underline{\gamma}\left(\mathcal{T}_{h}\right)$ on $\gamma_{E}$, as presented in [6] for a general setting, that is sufficient to show existence and uniqueness of the dG method. Therefore, we suppose from now on that the penalty parameters satisfy

$$
\gamma_{E} \geq \underline{\gamma}\left(\mathcal{T}_{h}\right) \quad \forall E \in \mathcal{E}_{h}
$$

Convergence properties of high-order $\mathrm{d} G$ methods are summarized in the following theorem, see e.g. [20].

Theorem 2. Let us suppose that $\Omega$ is a convex polygonal domain in $\mathbb{R}^{2}$ and that the solution to Problem (1) belongs to $H^{k+1}(\Omega)$. Let $\mathcal{T}_{h}, h>0$, be a family of matching shape regular triangular meshes on $\Omega$ and assume that parameters $\gamma_{E}$ satisfy the stability condition (28). Then, the following estimates hold for the $d G$ solution to (26) with a positive constant $C$ that depends only on the shape-regularity of $\mathcal{T}_{h}$ :

$$
\begin{aligned}
& \left\|u-u_{h}\right\|_{h} \leq C h^{k}\|u\|_{H^{k+1}(\Omega)} \\
& \left\|u-u_{h}\right\|_{L^{2}(\Omega)} \leq C h^{k+1}\|u\|_{H^{k+1}(\Omega)},
\end{aligned}
$$

where the $d G$ energy norm is defined on $\mathcal{V}_{h}^{k}$ as

$$
\left\|v_{h}\right\|\left\|_{h}^{2}=\sum_{T \in \mathcal{T}_{h}}\right\| \nabla v_{h}\left\|_{\left(L^{2}(K)\right)^{2}}^{2}+\sum_{E \in \mathcal{E}_{h}} \gamma_{E}\right\|\left[\left[v_{h}\right]\right] \|_{L^{2}(E)}^{2} .
$$

In the following sections, we will show that, depending on the approximation properties of the primal method and on the problem data, the order of the $\mathrm{dG}$ method, used to solve the dual problem, can be chosen in such a way that the respective error estimator is asymptotically exact. We will consider the following discretizations of the primal problem: high-order discontinuous Galerkin method, the first-order conforming finite element method, and the lowest order mixed Raviart-Thomas finite element method. Other non-conforming methods, finite volume and finite difference methods can be considered in a similar fashion using corresponding flux reconstruction algorithms, see e.g. [54, 29]. 


\section{Goal-oriented error estimates for dG methods}

We consider here the symmetric $\mathrm{dG}$ method of order $k \in \mathbb{N}$ for the discretization of Problem (1). We construct equilibrated fluxes $\mathbf{t}_{h}^{k-1}\left(u_{h}\right) \in$ $\mathbf{R T}_{k-1}$ in terms of the dG solution $u_{h} \in \mathcal{V}_{h}^{k}$, depending on the value of $k$, as follows (see e.g. [36, 21]):

$$
\begin{array}{r}
k \geq 1: \quad \int_{E} \mathbf{n}_{E} \cdot \mathbf{t}_{h}^{k-1}\left(u_{h}\right) q_{h}=\int_{E}\left(-\left\{\left\{\mathbf{n} \cdot D \nabla u_{h}\right\}\right\}+\gamma_{E}\left[\left[u_{h}\right]\right]\right) q_{h}, \\
\forall q_{h} \in \mathbb{P}_{k-1}(E), \forall E \in \mathcal{E}_{h} ; \\
k \geq 2: \quad \int_{T} \mathbf{t}_{h}^{k-1}\left(u_{h}\right) \cdot \mathbf{r}_{h}=-\int_{T} D \nabla u_{h} \cdot \mathbf{r}_{h} \\
+\sum_{E \in \partial T} \int_{E} \chi_{\mathcal{E}_{h}}(E) \mathbf{n} \cdot D \mathbf{r}_{h}\left[\left[u_{h}\right]\right], \\
\forall \mathbf{r}_{\mathbf{h}} \in\left(\mathbb{P}_{k-2}(T)\right)^{d}, \forall T \in \mathcal{T}_{h} .
\end{array}
$$

Note that the flux reconstruction using (31)-(32) is computationally inexpensive and is relatively easy to implement as an element-wise procedure.

Definition 3. A function $f \in L^{2}(\Omega)$ is said to have oscillations of order $l \in \mathbb{N}_{0}$ on $\mathcal{V}_{h}^{k}$ if there exists a constant $C$, that depends on $f$ and on the shape regularity of $\mathcal{T}_{h}$, such that

$$
\left\|f-\pi_{h}^{k} f\right\|_{L^{2}(\Omega)} \leq C h^{l} \quad \forall \mathcal{T}_{h}, h>0 .
$$

For example, it follows from the optimality of the orthogonal projection that a function $f \in H^{s}(\Omega)$ has oscillations of order $l=\min \{k+1, s\}$ on $\mathcal{V}_{h}^{k}$.

Theorem 3. Let $f \in L^{2}(\Omega)$ in Problem (1) have oscillations of order $k-1$ in $\mathcal{V}_{h}^{k-1}$. Let $u \in H^{k+1}(\Omega)$ and $u_{h} \in \mathcal{V}_{h}^{k}$ be the solutions to (1) and (26), respectively. Moreover, let $\mathbf{t}_{h}\left(u_{h}\right) \in \mathbf{R T}_{k-1}$ be the equilibrated flux with respect to $u_{h}$ given by (31)-(32). Then there exists a constant $C>0$, that depends on the problem data $\{D, f\}$ and on the shape-regularity of $\mathcal{T}_{h}$, such that

$$
\left\|\mathbf{t}_{h}^{k-1}\left(u_{h}\right)+D \nabla_{h} u_{h}\right\|_{\left[L^{2}(\Omega)\right]^{2}} \leq C h^{k} .
$$

Proof: Equality (17) follows from (31)-(32) and (26) by straightforward calculations. It is also known (see e.g. $[36,21])$ that the following estimate holds for $\mathbf{t}_{h}^{k-1}\left(u_{h}\right)$ given by (31)-(32):

$$
\left\|\mathbf{t}_{h}^{k-1}\left(u_{h}\right)+D \nabla_{h} u_{h}\right\|_{\left[L^{2}(\Omega)\right]^{2}} \leq\left\|u-u_{h}\right\|_{h}+h\left\|f-\pi_{h}^{k-1} f\right\|_{L^{2}(\Omega)} .
$$


Therefore, inequality (34) follows from above inequality owing to the a priori estimate (29) and oscillation estimate (33).

The next theorem provides the key result to investigate exactness of the error estimator (24) for the primal dG methods.

Theorem 4. Let $f \in L^{2}(\Omega)$ in Problem (1) have oscillations of order $k-1$ in $\mathcal{V}_{h}^{k-1}$ and let $u \in H^{k+1}(\Omega)$ be the solution to (1). Let $p \in H^{m+1}(\Omega)$ be the solution to the dual problem (7), with $m>k$, and suppose that the Riesz representer $q$ of the goal functional has oscillations of order $m-1$ in $\mathcal{V}_{h}^{m-1}$. Let $u_{h} \in \mathcal{V}_{h}^{k}$ and $p_{h} \in \mathcal{V}_{h}^{m}$ be the $d G$ finite element solutions to (26) and (27), respectively. Then, there exists a constant $C$, depending only on the shape regularity of $\mathcal{T}_{h}$, the data $\{D, f, q\}$ in the primal and dual problems, and the exact solutions $\{u, p\}$, such that

$$
\left|\mathcal{R}\left(u, p, f ; u_{h}, p_{h}\right)\right| \leq C h^{k+m} .
$$

Proof: Using the Cauchy-Schwarz inequality, the oscillation estimate for $f$, and the a priori estimate for the dual solution, there holds

$$
\begin{aligned}
\left|\sum_{T \in \mathcal{T}_{h}} \int_{T}\left(f-\pi_{h}^{k-1}(f)\right)\left(p-p_{h}\right)\right| & \leq\left\|f-\pi_{h}^{k-1}(f)\right\|_{L^{2}(\Omega)}\left\|p-p_{h}\right\|_{L^{2}(\Omega)} \\
& \leq C h^{k-1} h^{m+1}=C h^{k+m} .
\end{aligned}
$$

We infer from Theorem 3 that the reconstructed equilibrated flux $\mathbf{t}_{h}^{m-1}\left(p_{h}\right)$ with respect to the dual dG solution satisfies

$$
\left.\| \mathbf{t}_{h}^{m-1}\left(p_{h}\right)+D \nabla p\right) \|_{\left(L^{2}(\Omega)\right)^{2}} \leq C h^{m} .
$$

It follows that

$$
\begin{aligned}
& \left|\sum_{T \in \mathcal{T}_{h}} \int_{T}\left(\mathbf{t}_{h}^{k-1}\left(u_{h}\right)+D \nabla u_{h}\right) \cdot\left(\nabla p+D^{-1} \mathbf{t}_{h}^{m-1}\left(p_{h}\right)\right)\right| \\
& \quad \leq\left\|\mathbf{t}_{h}^{k-1}\left(u_{h}\right)+D \nabla u_{h}\right\|_{\left[L^{2}(\Omega)\right]^{2}} D^{-1}\left\|D \nabla p+\mathbf{t}_{h}^{m-1}\left(p_{h}\right)\right\|_{\left[L^{2}(\Omega)\right]^{2}} \\
& \quad \leq C h^{k} h^{m} .
\end{aligned}
$$


Now, owing to the smoothness of the exact solution and using the divergence theorem, one gets

$$
\begin{aligned}
\sum_{E \in \mathcal{E}_{h}} \int_{E}\left(\mathbf{n}_{E} \cdot\left(D \nabla p+\mathbf{t}_{h}^{m-1}\left(p_{h}\right)\right)\right)\left[\left[u_{h}\right]\right] \\
=\sum_{E \in \mathcal{E}_{h}} \int_{E}\left(\mathbf{n}_{E} \cdot\left(-D \nabla p-\mathbf{t}_{h}^{m-1}\left(p_{h}\right)\right)\right)\left[\left[u-u_{h}\right]\right] \\
=\sum_{T \in \mathcal{T}_{h}} \int_{T}\left(-D \nabla p-\mathbf{t}_{h}^{m-1}\left(p_{h}\right)\right) \cdot \nabla\left(u-u_{h}\right) \\
\quad+\sum_{T \in \mathcal{T}_{h}} \int_{T}\left(q-\pi_{h}^{m-1}(q)\right)\left(u-u_{h}\right) .
\end{aligned}
$$

Finally, using Theorem 3, the a priori estimates for the dG solutions, and the data oscillation estimate for the dual problem, one concludes that

$$
\left|\sum_{E \in \mathcal{E}_{h}} \int_{E}\left(\mathbf{n} \cdot\left(D \nabla p+\mathbf{t}_{h}^{m-1}\left(p_{h}\right)\right)\right)\left[\left[u_{h}\right]\right]\right| \leq C h^{k+m}
$$

which completes the proof.

Theorem 5. Let the conditions of Theorem 4 hold and let us suppose that the goal functional error is properly of order $h^{2 k}$. Then error estimator (21) is asymptotically exact, that is,

$$
\lim _{h \rightarrow 0^{+}} \frac{\eta\left(u_{h}, p_{h} ; \mathbf{t}_{h}^{k-1}\left(u_{h}\right), \mathbf{t}_{h}^{m-1}\left(p_{h}\right)\right)}{Q(u)-Q\left(u_{h}\right)}=1 .
$$

Proof: From Theorem 4, we infer that

$$
\left|\frac{\mathcal{R}\left(u, p, f ; u_{h}, p_{h}\right)}{Q(u)-Q\left(u_{h}\right)}\right| \leq c h^{m-k}
$$

with $m>k$, so that

$$
\lim _{h \rightarrow 0^{+}} \frac{\eta\left(u_{h}, p_{h} ; \mathbf{t}_{h}^{k-1}\left(u_{h}\right), \mathbf{t}_{h}^{m-1}\left(p_{h}\right)\right)}{Q(u)-Q\left(u_{h}\right)}=\lim _{h \rightarrow 0^{+}}\left[1-\frac{\mathcal{R}\left(u, p, f ; u_{h}, p_{h}\right)}{Q(u)-Q\left(u_{h}\right)}\right]=1 .
$$

Remark 4. The order doubling in the rate of convergence for the approximation of the goal functional is typical in the case of the symmetric version of the discontinuous Galerkin method, see e.g. [32]. 


\section{Goal-oriented error estimates for finite element methods}

We now consider the case in which Problem (1) is discretized by the lowest-order conforming finite element method:

$$
\text { Find } u_{h} \in V_{h} \text { such that } \int_{\Omega} D \nabla u_{h} \cdot \nabla v_{h}=\int_{\Omega} f v_{h}, \forall v_{h} \in V_{h},
$$

where $V_{h}=\mathcal{V}_{h}^{1} \cap H_{0}^{1}(\Omega)$. Note that the gradient $\nabla u_{h}$ is a piecewise constant vector field that may be discontinuous along the interelement edges. Following $[11,13]$, we consider reconstructed equilibrated fluxes in the form $\mathbf{t}_{h}\left(u_{h}\right)=-D \nabla u_{h}-\boldsymbol{\sigma}\left(u_{h}\right)$, where $\boldsymbol{\sigma}\left(u_{h}\right)$ represents some correction to the broken flux $D \nabla u_{h}$ such that $\mathbf{t}_{h}\left(u_{h}\right)$ belongs to $\mathbf{R T}_{0}\left(\mathcal{T}_{h}\right)$. Such a correction $\boldsymbol{\sigma}\left(u_{h}\right)$ is actually sought in the space

$$
\mathbf{R T}_{-1}\left(\mathcal{T}_{h}\right)=\left\{\mathbf{s}_{h} \in\left[L^{2}(\Omega)\right]^{2}:\left.\mathbf{s}_{h}\right|_{T} \in\left[\mathbb{P}_{0}(T)\right]^{2}+\mathbf{x} \mathbb{P}_{0}(T), \forall T \in \mathcal{T}_{h}\right\}
$$

and should satisfy the conditions

$$
\begin{aligned}
\operatorname{div} \boldsymbol{\sigma}\left(u_{h}\right) & =-\Pi_{0}(f), \\
{\left[\left[\boldsymbol{\sigma}\left(u_{h}\right) \cdot \mathbf{n}_{E}\right]\right] } & =-\left[\left[D \nabla u_{h} \cdot \mathbf{n}_{E}\right]\right] \quad \forall E \in \mathcal{E}_{h}^{\mathrm{i}},
\end{aligned}
$$

in order to guarantee that $\mathbf{t}_{h}\left(u_{h}\right)$ be a zero-order reconstructed flux. The existence of such $\boldsymbol{\sigma}\left(u_{h}\right)$ is proven in [13] in a more general situation and a constructive proof in the two-dimensional case is presented in [11]. The proof uses the hypercircle method for flux equilibration in the star patches $\omega_{i}$ associated with all vertices $i$ in $\mathcal{T}_{h}$. An estimate for this correction is provided in the following theorem (see [11], p. 184):

Theorem 6. There exists a constant $C>0$ that depends only on the shape regularity of $\mathcal{T}_{h}$ such that

$$
\left\|\boldsymbol{\sigma}\left(u_{h}\right)\right\|_{\left(L^{2}(\Omega)\right)^{2}} \leq C\left(\left\|\nabla u-\nabla u_{h}\right\|_{\left(L^{2}(\Omega)\right)^{2}}+h\left\|f-\pi_{h}^{0}(f)\right\|_{L^{2}(\Omega)}\right) .
$$

Theorem 7. Let $u \in H^{2}(\Omega)$ be the solution to (1) and let $p \in H^{3}(\Omega)$ be the solution to the dual problem (7). Suppose that the Riesz representer $q$ of the goal functional has first-order oscillations in $\mathcal{V}_{h}^{1}$. Let $u_{h} \in V_{h}$ be the finite element solution to (38) and $p_{h} \in \mathcal{V}_{h}^{2}$ be the second-order dual dG finite element solution to (27). Then, there exists a constant $C$, depending only on the shape regularity of $\mathcal{T}_{h}$, the data $\{D, f, q\}$ in the primal and dual problems, and the exact solutions $\{u, p\}$, such that

$$
\left|\mathcal{R}\left(u, p, f ; u_{h}, p_{h}\right)\right| \leq C h^{3} .
$$


Proof: The first term in (22) is estimated as

$$
\left|\sum_{T \in \mathcal{T}_{h}} \int_{T}\left(f-\pi_{h}^{0}(f)\right)\left(p-p_{h}\right)\right| \leq C h^{3},
$$

since, owing to the elliptic regularity, one has

$$
\left\|p-p_{h}\right\|_{L^{2}(\Omega)} \leq C h^{3} .
$$

From (36), (39), and an energy norm a priori error estimate for the finite element solution, we obtain by applying Cauchy-Schwarz:

$$
\begin{aligned}
\mid \sum_{T \in \mathcal{T}_{h}} \int_{T}\left(\mathbf{t}_{h}\left(u_{h}\right)\right. & \left.+D \nabla u_{h}\right) \cdot\left(\nabla p+D^{-1} \mathbf{t}_{h}^{1}\left(p_{h}\right)\right) \mid \\
= & \left|\sum_{T \in \mathcal{T}_{h}} \int_{T} \boldsymbol{\sigma}\left(u_{h}\right) \cdot\left(\nabla p+D^{-1} \mathbf{t}_{h}^{1}\left(p_{h}\right)\right)\right| \leq C h^{3} .
\end{aligned}
$$

This completes the proof since the third term in the remainder vanishes owing to the consistency of the method.

Theorem 8. Let $u_{h} \in V_{h}$ be the finite element solution to (38) and suppose that the goal functional error is properly of order $h^{2}$. Then, under the conditions of Theorem 7, the error estimator

$$
\begin{aligned}
\eta\left(u_{h}, p_{h} ; \mathbf{t}_{h}\left(u_{h}\right), \mathbf{t}_{h}^{1}\left(p_{h}\right)\right) & =\sum_{T \in \mathcal{T}_{h}} \int_{T}\left(f-\pi_{h}^{0}(f)\right) p_{h} \\
& -\sum_{T \in \mathcal{T}_{h}} \int_{T} \boldsymbol{\sigma}\left(u_{h}\right) \cdot D^{-1} \mathbf{t}_{h}^{1}\left(p_{h}\right)
\end{aligned}
$$

is asymptotically exact.

\section{Goal-oriented error estimates for the mixed $\mathbf{R T}_{0}$ finite element}

In this section, we consider the mixed form of the primal problem (1). The weak formulation then reads:

Find $(u, \mathbf{r}) \in L^{2}(\Omega) \times \mathbf{H}(\operatorname{div}, \Omega)$ such that

$$
\begin{array}{cc}
\int_{\Omega} D^{-1} \mathbf{r} \cdot \mathbf{q}-\int_{\Omega} u \nabla \cdot \mathbf{q}=0 & \forall \mathbf{q} \in \mathbf{H}(\operatorname{div}, \Omega) \\
\int_{\Omega} v \nabla \cdot \mathbf{r}=\int_{\Omega} f v & \forall v \in L^{2}(\Omega) .
\end{array}
$$


It is well known, see e.g. [15], that there exists a unique solution $(u, \mathbf{r})$ to the mixed problem and that the mixed formulation (42) is equivalent to (2) with $u \in H_{0}^{1}(\Omega)$ and $\mathbf{r}=-D \nabla u$. In addition, we have the following a priori estimates:

$$
\begin{aligned}
& \|u\|_{L^{2}(\Omega)} \leq C\|f\|_{L^{2}(\Omega)} \\
& \|\mathbf{r}\|_{\mathbf{H}(\operatorname{div}, \Omega)} \leq C\|f\|_{L^{2}(\Omega)}
\end{aligned}
$$

where constant $C$ depends only on parameter $D$ and domain $\Omega$. We introduce the finite element space:

$$
\mathbf{Q}_{0}\left(\mathcal{T}_{h}\right)=\left\{\mathbf{q}_{h} \in \mathbf{R T}_{0}\left(\mathcal{T}_{h}\right): \mathbf{q}_{h} \cdot \mathbf{n}=0 \text { on } \mathcal{E}_{h}^{\partial}\right\}
$$

The lowest-order Raviart-Thomas finite element method for the mixed formulation (42) reads:

Find $\left(u_{h}, \mathbf{r}_{h}\right) \in \mathbb{P}_{0} \times \mathbf{R T}_{0}\left(\mathcal{T}_{h}\right)$ such that

$$
\begin{array}{cc}
\int_{\Omega} D^{-1} \mathbf{r}_{h} \cdot \mathbf{q}_{h}-\int_{\Omega} u_{h} \nabla \cdot \mathbf{q}_{h}=0 & \forall \mathbf{q}_{h} \in \mathbf{Q}_{0}\left(\mathcal{T}_{h}\right) \\
\int_{\Omega} v_{h} \nabla \cdot \mathbf{r}_{h}=\int_{\Omega} f v_{h} & \forall v_{h} \in \mathbb{P}_{0} .
\end{array}
$$

Theorem 9. Let the Riesz representer $q$ of the goal functional have oscillations of first-order in $\mathcal{V}_{h}^{1}$. Let $\left(u_{h}, \mathbf{r}_{h}\right) \in \mathbb{P}_{0} \times \mathbf{R} \mathbf{T}_{0}\left(\mathcal{T}_{h}\right)$ be the solution to (44) and let $p_{h} \in \mathcal{V}_{h}^{2}$ be the second-order dual $d G$ finite element solution to (27). Then, there exists a constant $C$, depending only on the shape regularity of $\mathcal{T}_{h}$, the data $\{D, f, q\}$ in the primal and dual problems, and the exact solutions $\{(u, \mathbf{r}), p\}$, such that

$$
\left|\mathcal{R}\left((u, \mathbf{r}), D, f, q ;\left(u_{h}, \mathbf{r}_{h}\right), p_{h}\right)\right| \leq C h^{2} .
$$

Proof: The remainder (22) is estimated as follows. First, we determined the zeroth-order reconstructed flux $\mathbf{r}_{h}$ with respect to $u_{h}$. We then have

$$
\left|\sum_{T \in \mathcal{T}_{h}} \int_{T}\left(f-\pi_{h}^{0}(f)\right)\left(p-p_{h}\right)\right| \leq C h^{2},
$$

owing to the (suboptimal) estimate

$$
\left\|p-p_{h}\right\|_{L^{2}(\Omega)} \leq C h^{2}
$$


Next, from the elliptic regularity, we have $u \in H^{2}(\Omega)$ and $\mathbf{r} \in\left(H^{1}(\Omega)\right)^{2}$ so that the following a priori error estimates hold, see e.g. [15],

$$
\begin{aligned}
& \left\|\mathbf{r}-\mathbf{r}_{h}\right\|_{\left(L^{2}(\Omega)\right)^{2}} \leq C h\|\nabla \mathbf{r}\|_{\left(L^{2}(\Omega)\right)^{2}}, \\
& \left\|u-u_{h}\right\|_{L^{2}(\Omega)} \leq C h\left(\|\nabla \mathbf{r}\|_{\left(L^{2}(\Omega)\right)^{2}}+\|\nabla u\|_{\left(L^{2}(\Omega)\right)^{2}}\right) .
\end{aligned}
$$

Therefore

$$
\begin{aligned}
\left\|\mathbf{r}_{h}+D \nabla u_{h}\right\|_{\left(L^{2}(\Omega)\right)^{2}} & =\left\|\mathbf{r}_{h}\right\|_{\left(L^{2}(\Omega)\right)^{2}} \\
& \leq\left\|\mathbf{r}-\mathbf{r}_{h}\right\|_{\left(L^{2}(\Omega)\right)^{2}}+\|\mathbf{r}\|_{\left(L^{2}(\Omega)\right)^{2}} \\
& \leq C(1+h)\|\mathbf{r}\|_{\left(L^{2}(\Omega)\right)^{2}},
\end{aligned}
$$

which yields

$$
\left|\sum_{T \in \mathcal{T}_{h}} \int_{T}\left(\mathbf{r}_{h}+D \nabla u_{h}\right) \cdot\left(\nabla p+D^{-1} \mathbf{t}_{h}^{1}\left(p_{h}\right)\right)\right| \leq C h^{2} .
$$

Noting that

$$
\begin{aligned}
& \left|\sum_{E \in \mathcal{E}_{h}} \int_{E} \mathbf{n}_{E} \cdot\left(D \nabla p+\mathbf{t}_{h}^{1}\left(p_{h}\right)\right)\left[\left[u_{h}\right]\right]\right| \\
& \quad \leq\left|\sum_{T \in \mathcal{T}_{h}} \int_{T}\left(D \nabla p+\mathbf{t}_{h}^{1}\left(p_{h}\right)\right) \cdot \nabla u\right|+\left|\sum_{T \in \mathcal{T}_{h}} \int_{T}\left(q-\pi_{h}^{1}(q)\right)\left(u-u_{h}\right)\right| \\
& \quad \leq C h^{2}\|\nabla u\|_{\left(L^{2}(\Omega)\right)^{2}}+C h\left\|u-u^{h}\right\|_{L^{2}(\Omega)} \leq C h^{2}
\end{aligned}
$$

allows us to conclude.

Theorem 10. Let $\left(u_{h}, \mathbf{r}_{h}\right) \in \mathbb{P}_{0} \times \mathbf{R} \mathbf{T}_{0}\left(\mathcal{T}_{h}\right)$ be the solution to (44) and let the error in the goal functional be assumed properly of first order. Then, under the conditions of Theorem 9, the error estimator

$$
\begin{aligned}
\eta\left(u_{h}, p_{h} ; \mathbf{r}_{h}, \mathbf{t}_{h}^{1}\left(p_{h}\right)\right) & =\sum_{T \in \mathcal{T}_{h}} \int_{T}\left(f-\pi_{h}^{0}(f)\right) p_{h} \\
& +\sum_{T \in \mathcal{T}_{h}} \int_{T} \mathbf{r}_{h} \cdot D^{-1} \mathbf{t}_{h}^{1}\left(p_{h}\right)-\sum_{E \in \mathcal{E}_{h}} \int_{E} \mathbf{n}_{E} \cdot \mathbf{t}_{h}^{1}\left(p_{h}\right)\left[\left[u_{h}\right]\right]
\end{aligned}
$$

is asymptotically exact. 


\section{Numerical examples}

We shall consider two classes of benchmark problems: one with large data oscillations and one with no data oscillation. The first problem, following [19], is the homogeneous boundary-value problem (1) with $D=1$ in $\Omega=(0,1)^{2}$, for which the loading term $f$ is given such that the exact solution reads

$$
u(x, y)=10^{4} x(1-x) y(1-y) \exp \left(-100\left((x-0.75)^{2}+(y-0.75)^{2}\right)\right) .
$$

The goal functional is chosen for the first problem as:

$$
Q(u)=\frac{1}{|\omega|} \int_{\omega} u
$$

with

$$
\omega=\{(x, y) \in \Omega: 1.5 \leq x+y \leq 1.75\} .
$$

In other words, the goal is to calculate the average of the solution over the strip $\omega$, namely the region near the maximum of the solution, where the righthand side $f$, the solution $u$, and the gradient of $u$ exhibit large oscillations. The exact value of the quantity of interest was evaluated analytically to be $Q(u)=43.28448988$.

The second benchmark problem is given by the homogeneous boundaryvalue problem (1) in $\Omega=(0,1)^{2}$ with uniform load $f=10^{3}$, for which the oscillations vanish. The goal functional is given by (50) evaluated over the subdomain

$$
\omega=[0.75,1] \times[0.75,1],
$$

which corresponds to the averaged solution in the upper-right corner where the solution exhibits large gradient. A representation of the exact solution in terms of series was used to calculate the exact value of the functional.

The objective of the numerical experiments is to study the behavior of the contributions to the error estimator $\eta(21)$ in the case of three finite element methods described above. For each problem, we describe the numerical results that confirm the asymptotical exactness of the suggested error estimator and demonstrate the potential of such an estimator in goal-oriented mesh adaptation. In all examples presented below, meshes are adapted using a refinement strategy based on the method proposed by Dörfler [23], whereby the elements in a minimal set $\mathcal{M} \subset \mathcal{T}_{h}$, such that

$$
\theta \sum_{T \in \mathcal{M}} \eta(T)^{2} \leq \sum_{T \in \mathcal{T}_{h}} \eta(T)^{2}
$$


are refined. The refinement parameter $\theta=0.75$ was used in the first benchmark problem while $\theta=0.5$ was considered in the second problem. Elements are refined using the longest edge bisection technique and additional refinements of the mesh are considered in order to eliminate hanging nodes.

Finally, the quality of the error estimator $\eta=\eta\left(u_{h}, p_{h} ; \mathbf{t}_{h}\left(u_{h}\right), \mathbf{t}_{h}\left(p_{h}\right)\right)$ will be assessed in terms of the effectivity index:

$$
\mathcal{I}_{\eta}=\frac{\eta\left(u_{h}, p_{h} ; \mathbf{t}_{h}\left(u_{h}\right), \mathbf{t}_{h}\left(p_{h}\right)\right)}{Q(u)-Q\left(u_{h}\right)}
$$

evaluated on sequences of uniformly and adaptively refined meshes.

\section{1. $d G$ method}

We consider here the first-order and second-order symmetric dG methods for the discretization of the primal and dual problems and reconstruct the equilibrated fluxes in $\mathbf{R T}_{0}$ and $\mathbf{R} \mathbf{T}_{1}$, respectively.

We show in Figure 1(a) the effectivity index of estimator $\eta$ for a sequence of uniform and adaptive mesh refinements for the first test problem. For uniform refinement, the effectivity index converges to unity as the mesh size decreases, which confirms asymptotic exactness of the error estimator. In Figure 1(b) the true error $\mathcal{E}$ in the goal functional approximation as well as the error estimator and its different components, are shown for a sequence of adaptive mesh refinements. To illustrate the effect of error cancelation when summing up the three contributions, the positive and negative parts of each component are shown in black and red, respectively. Moreover, given a quantity $f$ (i.e. $e$ or $\eta$ ), we denote by $f^{+}=\max \{f, 0\}$ (resp. $f^{-}=\max \{-f, 0\}$ ) the positive (resp. negative) part of $f$. We can see that the error estimate remains very close to the true error offering accurate information for mesh adaptation. For such a problem with large data oscillations, the error estimate is essentially quantified by the difference between the oscillation contribution $\eta_{\mathcal{O}}$ and the flux contribution $\eta_{\nabla}$, which both dominate in absolute value but with opposite sign, and is corrected by the relatively smaller $\eta_{\mathcal{H}}$ component.

In the case of the second test problem, the effectivity index is shown in Figure (2)(a) for uniform and adapted sequences of meshes and the exact error $e$, error estimator $\eta$ and its components $\eta_{\mathcal{O}}, \eta_{\nabla}$, and $\eta_{\mathcal{H}}$ are shown in Figure (2)(b). In the absence of oscillations in the problem data, the component $\eta_{\mathcal{O}} \simeq 0$ (within machine precision) as expected, and the flux error estimate $\eta_{\nabla}$ is the dominant contribution to the error estimator. 

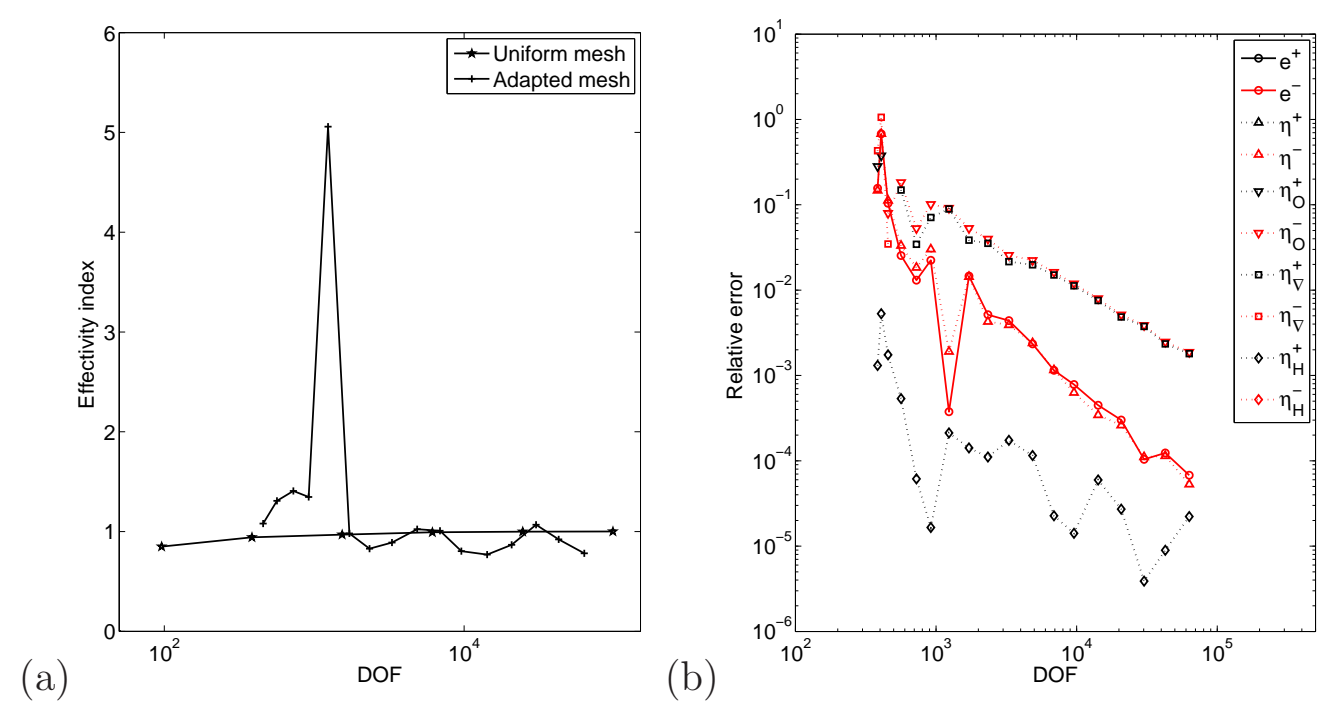

Figure 1: First benchmark problem using the dG method for the solution of the primal problem: (a) effectivity index $\mathcal{I}_{\eta}$; (b) exact error $e$ in quantity of interest, error estimator $\eta$, and contributions $\eta_{\mathcal{O}}, \eta_{\nabla}$, and $\eta_{\mathcal{H}}$ to error estimate $\eta$ on a sequence of adapted meshes (negative values are shown in red, positive values in black).

\subsection{Conforming finite element method}

In the case of the first test problem, we consider the first-order conforming finite element method for the primal problem and dG methods of various orders for the dual problem. The equilibrated fluxes for the primal problem was constructed using the hypercircle method presented in [11]. In Figure 3(a), we present the effectivity index of estimator $\eta$ for a sequence of uniform and adaptive mesh refinements. In the case of uniform refinements, we use $d G$ methods of order first, second, and third for the calculation of the error estimates. As one can observe from the figure, the first-order dG method does not provide an asymptotically exact error estimator (the effectivity index tends to $\approx 1.9$ on fine meshes), but asymptotic exactness is recovered in accordance with Theorem 8 in the case of the second- and third-order dG methods. We show in Figure 3(b) the exact error $e$ in the goal functional approximation as well as the error estimate $\eta$ and the different contributions (calculated using the second-order $\mathrm{dG}$ method) for a sequence of adaptive mesh refinements. The component $\eta_{\mathcal{H}}$ vanishes here, so the difference between the oscillation estimate $\eta_{\mathcal{O}}$ and the flux estimate $\eta_{\nabla}$, which are of the same order but of opposite signs, accurately captures the goal functional 

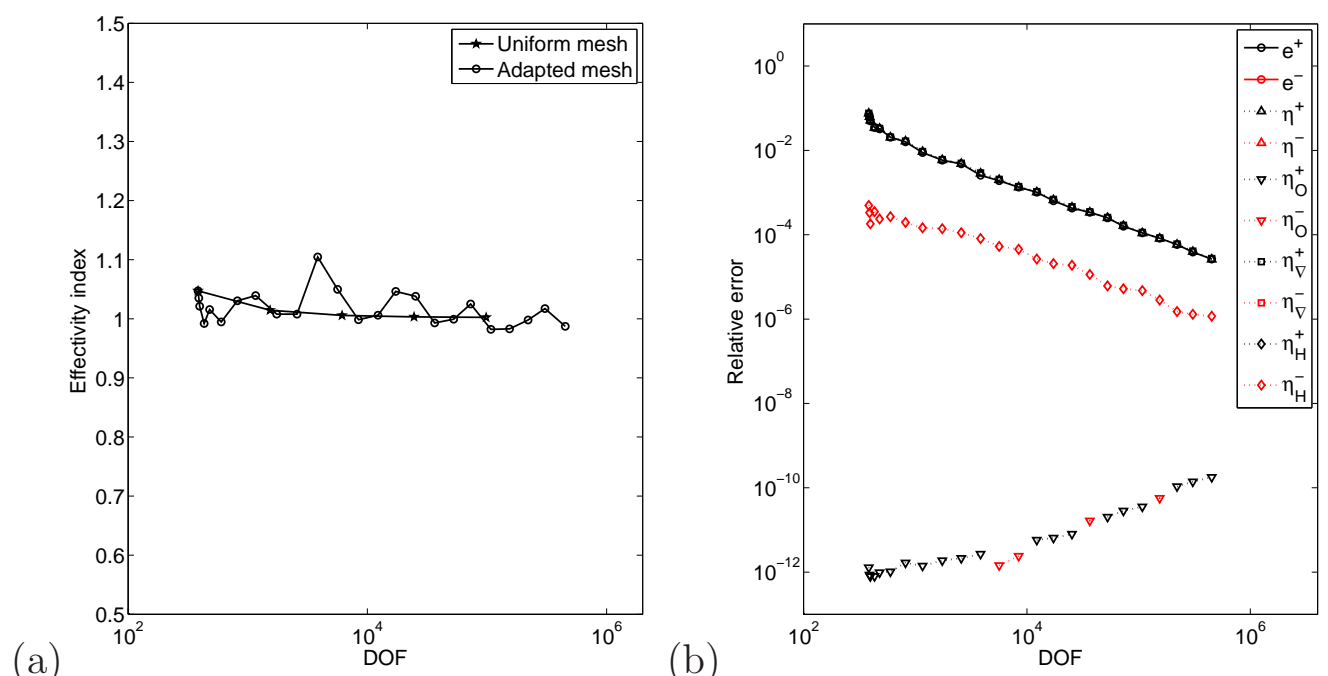

Figure 2: Second benchmark problem using the dG method for the solution of the primal problem: (a) effectivity index $\mathcal{I}_{\eta}$; (b) exact error $e$ in quantity of interest, error estimator $\eta$, and contributions $\eta_{\mathcal{O}}, \eta_{\nabla}$, and $\eta_{\mathcal{H}}$ to error estimate $\eta$ on a sequence of adapted meshes (negative values are shown in red, positive values in black).

error.

In the case of the second benchmark problem, we use the same finite element method for the primal problem as before and restrict ourselves to the second-order symmetric dG method for the dual problem. Flux reconstruction is performed as in the previous test problem. The numerical results, shown in Figure (4), are as expected since in this case both $\eta_{\mathcal{O}} \approx 0$ and $\eta_{\mathcal{H}} \approx 0$.

\subsection{Raviart-Thomas mixed finite element}

We solve the first benchmark problem using $\mathbf{R} \mathbf{T}_{0}$ mixed finite elements, calculate the error estimate (48) on a sequence of uniform meshes using the first- and second-order symmetric $\mathrm{dG}$ methods, and reconstruct equilibrated fluxes in $\mathbf{R T}_{0}$ and $\mathbf{R} \mathbf{T}_{1}$, respectively. The results are shown in Figure 5(a). Theorem 10 is not valid in the first case and we can see that the effectivity index fails to converge to unity as expected. When the approximation order of both the dG method used for the solution of the dual problem and flux reconstruction method increases, the error estimate becomes again asymptotically exact. The exact error and the error indicator with its components, calcu- 

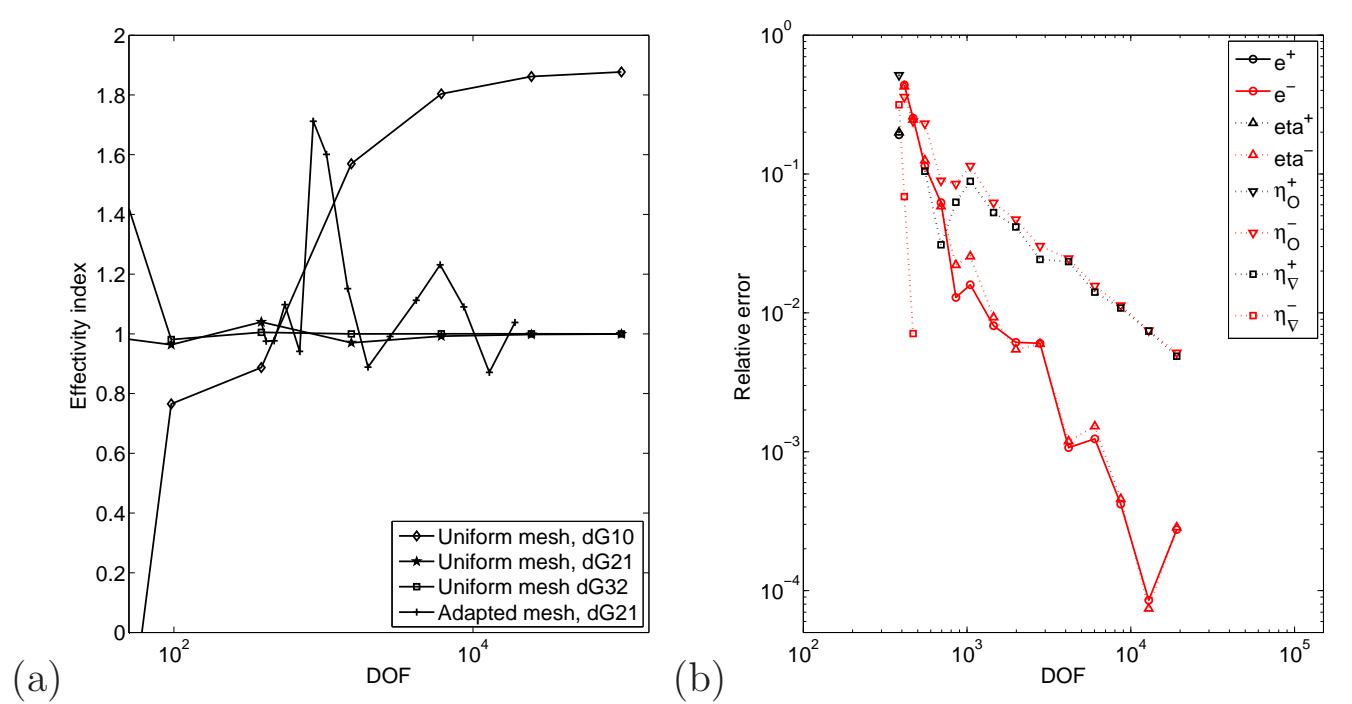

Figure 3: First benchmark problem using the conforming finite element method for the solution of the primal problem: (a) effectivity index $\mathcal{I}_{\eta}$; (b) exact error $e$ in quantity of interest, error estimator $\eta$, and contributions $\eta_{\mathcal{O}}, \eta_{\nabla}$, and $\eta_{\mathcal{H}}$ to error estimate $\eta$ on a sequence of adapted meshes (negative values are shown in red, positive values in black).

lated using the second-order symmetric $\mathrm{dG}$ method and equilibrated fluxes reconstructed in $\mathbf{R T}_{1}$, are shown in Figure $5(\mathrm{~b})$ on a sequence of adapted meshes. The flux estimate $\eta_{\nabla}$ and jump estimate $\eta_{\mathcal{H}}$ are in this case very close to each other in absolute value (of the order $\mathcal{O}(1)$ ) but are of opposite signs. It is interesting to see that the error $e$ is essentially driven here by the oscillation error $\eta_{\mathcal{O}}$.

The second problem is solved using $\mathbf{R T}_{0}$ mixed finite elements as before. Here, the error estimate (48) is also evaluated using the second-order symmetric dG method and reconstructed fluxes in $\mathbf{R T}_{1}$. The results are presented in Figure 6 . In absence of data oscillations, $\eta_{O} O \approx 0\left(\eta_{O} O\right.$ is actually not shown in Figure 6(b)), and the error estimate $\eta$ amounts to the the difference between the flux and the jump estimates. Even in this case, we can observe that the error estimate $\eta$ is asymptotically exact as demonstrated in Figure 6(a). 

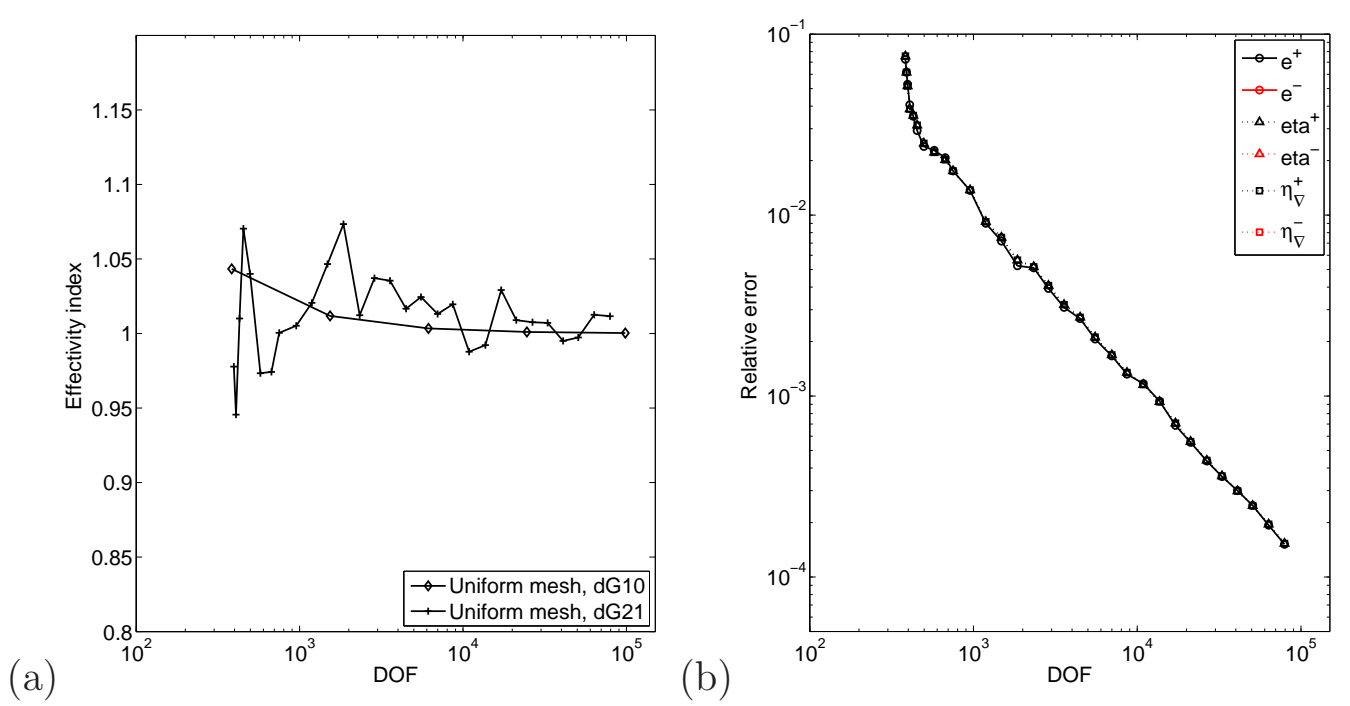

Figure 4: Second benchmark problem using the conforming finite element method for the solution of the primal problem: (a) effectivity index $\mathcal{I}_{\eta}$; (b) exact error $e$ in quantity of interest, error estimator $\eta$, and contributions $\eta_{\mathcal{O}}, \eta_{\nabla}$, and $\eta_{\mathcal{H}}$ to error estimate $\eta$ on a sequence of adapted meshes (negative values are shown in red, positive values in black).

\section{Conclusions}

We have presented a flexible approach for the computation of a posteriori error estimates with respect to quantities of interest in the case of elliptic problems. The main ingredients of the method are the calculation of higherorder approximation of the dual problem by the discontinuous Galerkin method and the construction of equilibrated fluxes in Raviart-Thomas finite element spaces. The advantage of using a dG method for the dual problem lies in its simplicity to consider high-order approximations and to reconstruct equilibrated fluxes from its solution. The error estimator in the quantity of interest is decomposed into three contributions: 1) an error estimate due to the data oscillations in the primal problem, 2) an error estimate that measures the difference between the reconstructed fluxes and the finite element fluxes, and 3) a contribution from the jump of the finite element solution at the interface of the elements, all terms being weighted by the discontinuous Galerkin solution to the dual problem. Within this framework, the primal problem can be approximated by any finite element method as long as it satisfies standard a priori error estimates and its solution can be subjected to 

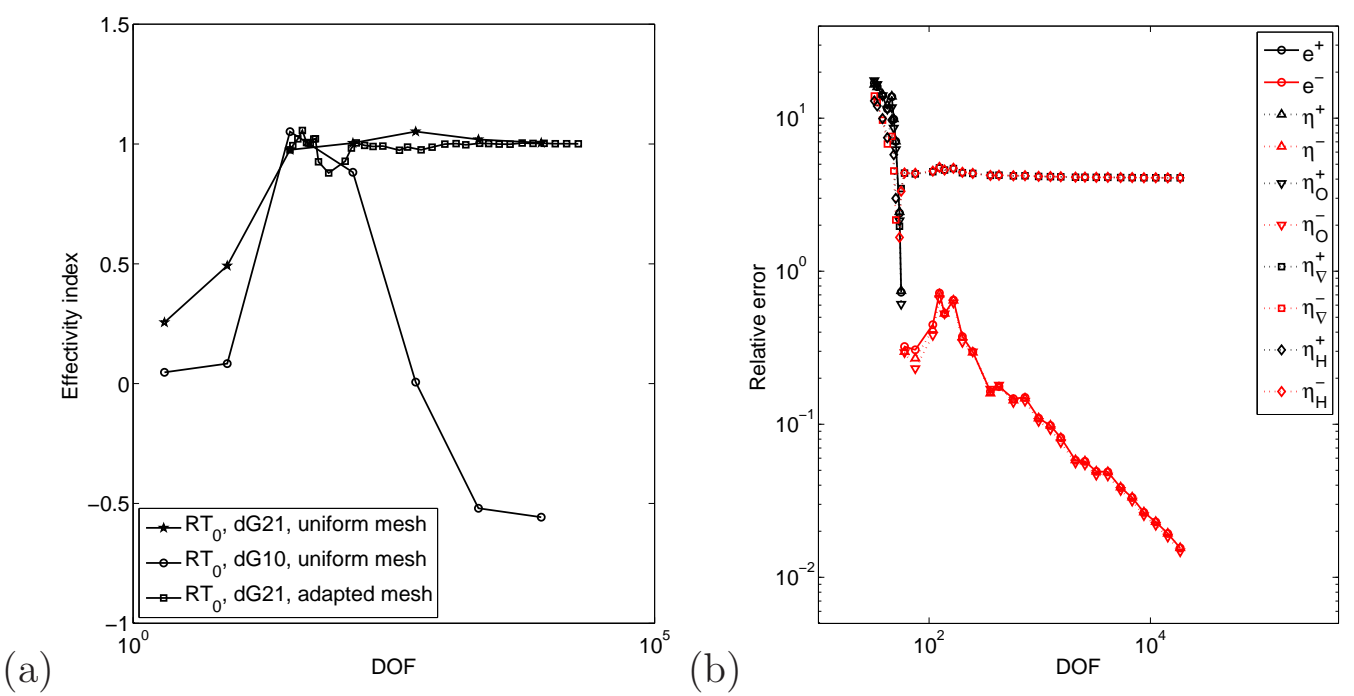

Figure 5: First benchmark problem using the mixed $\mathbf{R} \mathbf{T}_{0}$ method for the solution of the primal problem and dG method for the dual problem: (a) effectivity index $\mathcal{I}_{\eta}$; (b) exact error $e$ in quantity of interest, error estimator $\eta$, and contributions $\eta_{\mathcal{O}}, \eta_{\nabla}$, and $\eta_{\mathcal{H}}$ to error estimate $\eta$ on a sequence of adapted meshes (negative values are shown in red, positive values in black).

the reconstruction of equilibrated fluxes. We have shown that, depending on the convergence properties of the underlying method, the order of the dual $\mathrm{dG}$ method can be chosen in such a way that the resulting error estimator be asymptotically exact. Numerical experiments, using either the $\mathrm{dG}$ method, the conforming finite element method, or the mixed Raviart-Thomas method for the solution of the primal problem, clearly confirm that the proposed error estimator has effectivity indices close to unity and is asymptotically exact.

\section{Acknowledgements}

The first author gratefully acknowledges the partial support by CNPq, Brazil. The second author is grateful for the support by a Discovery Grant from the Natural Sciences and Engineering Research Council of Canada. He is also a participant of the KAUST SRI center for Uncertainty Quantification in Computational Science and Engineering. 

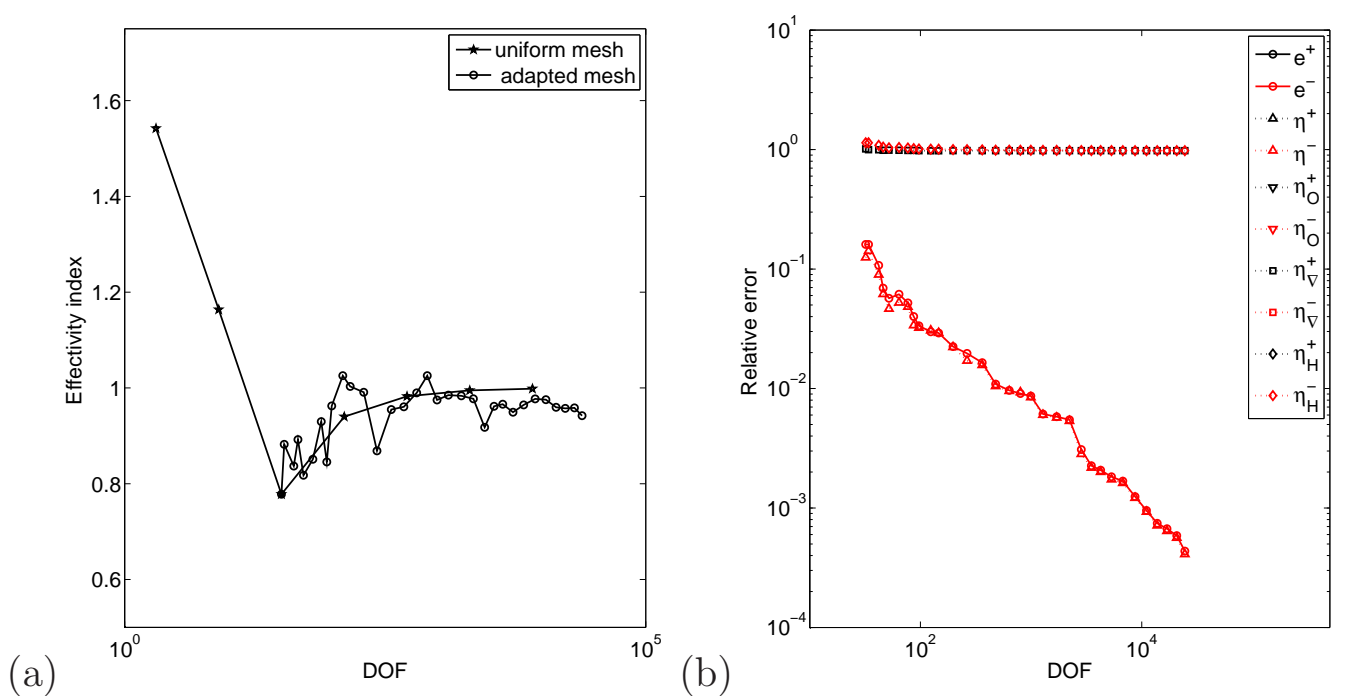

Figure 6: Second benchmark problem using the mixed $\mathbf{R T}_{0}$ method for the solution of the primal problem and $\mathrm{dG}$ method for the dual problem: (a) effectivity index $\mathcal{I}_{\eta}$; (b) exact error $e$ in quantity of interest, error estimator $\eta$, and contributions $\eta_{\mathcal{O}}, \eta_{\nabla}$, and $\eta_{\mathcal{H}}$ to error estimate $\eta$ on a sequence of adapted meshes (negative values are shown in red, positive values in black).

\section{References}

[1] M. Ainsworth. A posteriori error estimation for discontinuous Galerkin finite element approximation. SIAM J. Numer. Anal., 45(4):1777-1798, 2007.

[2] M. Ainsworth. A posteriori error estimation for lowest order RaviartThomas mixed finite elements. SIAM J. Sci. Comput., 30(1):189-204, 2008.

[3] M. Ainsworth. A framework for obtaining guaranteed error bounds for finite element approximations. J. Comput. Appl. Math., 234(9):2618$2632,2010$.

[4] M. Ainsworth and J. T. Oden. A posteriori error estimation in finite element analysis. John Wiley \& Sons, 2000.

[5] M. Ainsworth and R. Rankin. Guaranteed computable bounds on quan- 
tities of interest in finite element computations. Internat. J. Numer. Methods Engrg., 89(13):1605-1634, 2012.

[6] M. Ainsworth and R. Rankin. Technical note: A note on the selection of the penalty parameter for discontinuous Galerkin finite element schemes. Numer. Methods Partial Differential Equations, 28(3):1099-1104, 2012.

[7] I. Babuška and T. Strouboulis. The Finite Element Method and its Reliability. Oxford University Press, New York, 2001.

[8] W. Bangerth and R. Rannacher. Adaptive Finite Element Methods for Differential Equations. Birkhäuser Verlag, Basel, 2003.

[9] R. Becker, P. Hansbo, and M. G. Larson. Energy norm a posteriori error estimation for discontinuous Galerkin methods. Comput. Methods Appl. Mech. Engrg., 192(5-6):723-733, 2003.

[10] R. Becker and R. Rannacher. An optimal control approach to a posteriori error estimation in finite element methods. Acta Numer., 10:1-102, 2001.

[11] D. Braess. Finite Elements. Theory, Fast Solvers, and Application in Solid Mechanics. Cambridge University Press, 2007.

[12] D. Braess. An equilibrated a posteriori error estimator fort he interior penalty discontinuous Galerkin method. Preprint 08/2013, Institut für Mathematik, Universität Augsburg, April 2013.

[13] D. Braess and J. Schöberl. Equilibrated residual error estimator for edge elements. Math. Comp., 77(262):651-672, 2008.

[14] S. C. Brenner and L. R. Scott. The Mathematical Theory of Finite Element Methods. Springer, 1994.

[15] F. Brezzi and M. Fortin. Mixed and Hybrid Finite Element Methods, volume 15 of Springer Series in Computational Mathematics. SpringerVerlag, New York, 1991.

[16] C. Carstensen. A posteriori error estimate for the mixed finite element method. Math. Comp., 66(218):465-476, 1997. 
[17] C. Carstensen. Lectures on adaptive mixed finite element methods. In Mixed finite element technologies, volume 509 of CISM Courses and Lectures, pages 1-56. Springer, 2009.

[18] C. Carstensen, T. Gudi, and M. Jensen. A unifying theory of a posteriori error control for discontinuous Galerkin FEM. Numer. Math., 112(3):363-379, 2009.

[19] C. Carstensen and C. Merdon. Effective postprocessing for equilibration a posteriori error estimators. Numer. Math., 123(3):425-459, 2013.

[20] D. A. Di Pietro and A. Ern. Mathematical Aspects of Discontinuous Galerkin Methods, volume 69 of Mathématiques \&s Applications. Springer, 2011.

[21] D. A. Di Pietro and A. Ern. Analysis of a discontinuous Galerkin method for heterogeneous diffusion problems with low-regularity solutions. $\mathrm{Nu}$ mer. Methods Partial Differ. Equations, 28:1161-1171, 2012.

[22] V. Dolejší, A. Ern, and M. Vohralík. A Framework for Robust A Posteriori Error Control in Unsteady Nonlinear Advection-Diffusion Problems. SIAM J. Numer. Anal., 51(2):773-793, 2013.

[23] W. Dörfler. A convergent adaptive algorithm for Poisson's equation. SIAM J. Numer. Anal., 33(3):1106-1124, 1996.

[24] L. El Alaoui, A. Ern, and M. Vohralík. Guaranteed and robust a posteriori error estimates and balancing discretization and linearization errors for monotone nonlinear problems. Comput. Methods Appl. Mech. Engrg., 200(37-40):2782-2795, 2011.

[25] K. Eriksson, D. Estep, P. Hansbo, and C. Johnson. Introduction to adaptive methods for differential equations. In Introduction to adaptive methods for differential equations, Acta Numer., pages 105-158. Cambridge University Press, Cambridge, 1995.

[26] A. Ern, S. Nicaise, and M. Vohralík. An accurate H(div) flux reconstruction for discontinuous Galerkin approximations of elliptic problems. $C$. R. Math. Acad. Sci. Paris, 345(12):709-712, 2007. 
[27] A. Ern, A. F. Stephansen, and M. Vohralík. Guaranteed and robust discontinuous Galerkin a posteriori error estimates for convection-diffusionreaction problems. J. Comput. Appl. Math., 234(1):114-130, 2010.

[28] A. Ern and M. Vohralík. A unified framework for a posteriori error estimation in elliptic and parabolic problems with application to finite volumes. In Finite volumes for complex applications. VI. Problems 8 perspectives. Volume 1, 2, volume 4 of Springer Proc. Math., pages 821837. Springer, Heidelberg, 2011.

[29] A. Ern and M. Vohralík. Four closely related equilibrated flux reconstructions for nonconforming finite elements. C. R. Math. Acad. Sci. Paris, 351(1-2):77-80, 2013.

[30] P. Grisvard. Singularities in Boundary Value Problems. Research Notes in Applied Mathematics. Masson, Paris, 1992.

[31] W. Han. A posteriori error analysis via duality theory, volume 8 of Advances in Mechanics and Mathematics. Springer-Verlag, 2005.

[32] K. Harriman, P. Houston, B. Senior, and E. Süli. hp-version discontinuous Galerkin methods with interior penalty for partial differential equations with nonnegative characteristic form. In Recent advances in scientific computing and partial differential equations (Hong Kong, 2002), volume 330 of Contemp. Math., pages 89-119. Amer. Math. Soc., Providence, RI, 2003.

[33] P. Houston, D. Schötzau, and T. P. Wihler. Energy norm a posteriori error estimation of $h p$-adaptive discontinuous Galerkin methods for elliptic problems. Math. Models Methods Appl. Sci., 17(1):33-62, 2007.

[34] O. A. Karakashian and F. Pascal. A posteriori error estimates for a discontinuous Galerkin approximation of second-order elliptic problems. SIAM J. Numer. Anal., 41(6):2374-2399, 2003.

[35] K. Y. Kim. A posteriori error analysis for locally conservative mixed methods. Math. Comp., 76(257):43-66, 2007.

[36] K. Y. Kim. A posteriori error estimators for locally conservative methods of nonlinear elliptic problems. Appl. Numer. Math., 57(9):1065-1080, 2007. 
[37] K.-Y. Kim. Guaranteed a posteriori error estimator for mixed finite element methods of elliptic problems. Appl. Math. Comput., 218(24):11820-11831, 2012.

[38] S. Korotov. A posteriori error estimation of goal-oriented quantities for elliptic type BVPs. J. Comput. Appl. Math., 191(2):216-227, 2006.

[39] S. Korotov, P. Neittaanmäki, and S. Repin. A posteriori error estimation of goal-oriented quantities by the superconvergence patch recovery. $J$. Numer. Math., 11(1):33-59, 2003.

[40] D. Kuzmin and S. Korotov. Goal-oriented a posteriori error estimates for transport problems. Math. Comput. Simulation, 80(8):1674-1683, 2010 .

[41] R. Lazarov, S. Repin, and S. K. Tomar. Functional a posteriori error estimates for discontinuous Galerkin approximations of elliptic problems. Numer. Methods Partial Differ. Equations, 25(4):952-971, 2009.

[42] C. Lovadina and L. D. Marini. A-posteriori error estimates for discontinuous Galerkin approximations of second order elliptic problems. J. Sci. Comput., 40(1-3):340-359, 2009.

[43] P. Neittaanmaki and S. Repin. Reliable methods for computer simulation, volume 33 of Studies in Mathematics and its Applications, chapter Error control and a posteriori estimates. Elsevier, Amsterdam, 2004.

[44] P. Neittaanmäki, S. Repin, and P. Turchyn. New a posteriori error indicator in terms of linear functionals for linear elliptic problems. Russian J. Numer. Anal. Math. Modelling, 23(1):77-87, 2008.

[45] J. T. Oden and S. Prudhomme. Goal-oriented error estimation and adaptivity for the finite element method. Comput. Math. Appl., 41(56):735-756, 2001.

[46] J. T. Oden and S. Prudhomme. Estimation of modeling error in computational mechanics. Journal of Computational Physics, 182:496-515, 2002.

[47] J. S. Ovall. Asymptotically exact functional error estimators based on superconvergent gradient recovery. Numer. Math., 102(3):543-558, 2006. 
[48] S. Prudhomme and J. T. Oden. On goal-oriented error estimation for elliptic problems: application to the control of pointwise errors. Comput. Methods Appl. Mech. Engrg., 176(1-4):313-331, 1999.

[49] S. Prudhomme and J. T. Oden. Computable error estimators and adaptive techniques for fluid flow problems. In Error estimation and adaptive discretization methods in computational fluid dynamics, volume 25 of Lect. Notes Comput. Sci. Eng., pages 207-268. Springer, Berlin, 2003.

[50] S. I. Repin. A Posteriori Estimates for Partial Differential Equations, volume 4 of Radon Series on Computational and Applied Mathematics. Walter de Gruyter GmbH \& Co. KG, 2008.

[51] R. Schneider, Y. Xu, and A. Zhou. An analysis of discontinuous Galerkin methods for elliptic problems. Adv. Comput. Math., 25(1-3):259-286, 2006.

[52] R. Verfürth. A Review of A Posteriori Estimation and Adaptive Mesh Refinement Techniques. Wiley-Teubner, New York, 1996.

[53] R. Verfürth. A posteriori error estimation techniques for finite element methods. Numerical Mathematics and Scientific Computation. Oxford University Press, Oxford, 2013.

[54] M. Vohralík. Guaranteed and fully robust a posteriori error estimates for conforming discretizations of diffusion problems with discontinuous coefficients. J. Sci. Comput., 46(3):397-438, 2011.

[55] J. M. Yang and Y. P. Chen. A unified a posteriori error analysis for discontinuous Galerkin approximations of reactive transport equations. J. Comput. Math., 24(3):425-434, 2006. 